channels. The only possibility for a channel to be made available to waiting handover requests in a cell comes when a channel is released after natural termination of a call, or after a handover request from a mobile of the current cell either succeeds or fails. However, it is possible for a mobile which has requested handover to an adjacent cell to exchange its channel with that of another mobile, if there are any, moving from that cell to the current cell. Thus, both the mobiles will be successfully handed over without any channel being explicitly released. This is the basis of the new channel exchange scheme presented in this Letter.

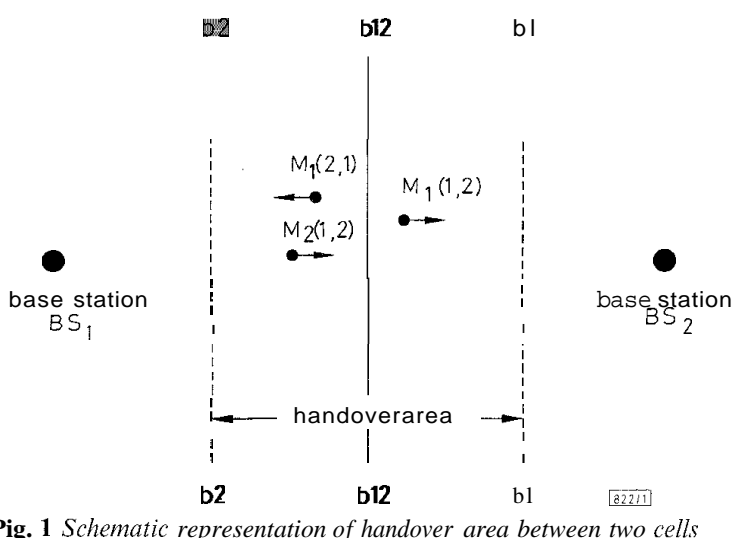

The new scheme: Consider the handover region between two cells in a mobile cellular communication system as shown in Fig. 1. Here, b12 is the physical boundary line. The lines b1 and b2 are the radio coverage boundaries of base stations $\mathrm{BS}$, and $\mathrm{BS}$, respectively. For successful handover, a mobile has to be allocated a new channel from the target cell while it is moving between the lines bl and b2. In this Letter, a mobile in the handover area is denoted by $\mathbf{M}_{i}(j, k)$, which represents the ith mobile holding a channel belonging to cellj and moving towards the cell $k$. In the conventional channel allocation scheme, mobile $\mathrm{M}_{i}(j, k)$ has to wait until a free channel is available with $\mathrm{BS}_{k}$. In the new scheme, however, the mobiles are allowed to exchange their channels if they are moving in opposite directions in the handover area. Thus, with the situation shown in Fig. 2, the channels held by the mobiles $M_{1}(1,2)$ and $M_{1}(2,1)$ are exchanged. The new scheme requires a close interaction between neighbouring base stations. A base station BS, enqueues a handover request from a mobile moving from cell $j$ to cell $i$ in a separate queue $Q,$. Similarly, the base station BS, maintains a queue $\mathrm{Q}_{j i}$ for handover requests from mobiles moving from cell $i$ to cell $j$. The two base stations BS, and $\mathrm{BS}$, together process the queues $\mathrm{Q}$, and $\mathrm{Q}_{i}$ in order to allow the exchange of channels for suitably prioritised entries in the queues. The prioritisation of the enqueued handover requests can follow any of the reported schemes, such as those given in [2 -4].

A handover request from a mobile in cell $i$ to the base station of cell $j$ is processed in any of the following three ways:

\title{
Handover scheme for mobile cellular communication systems
}

\author{
D.K. Anvekar and S.S. Pradhan
}

Indexing terms: Cellular radio, Mobile communication systems

A new scheme for minimising handover failure probability in mobile cellular communication systems, by exchanging channels between two mobiles moving in opposite directions across the handover area of adjacent cells, is presented. The performance evaluation of the new scheme is carried out by computer simulation of a two cell model.

Introduction: Minimising handover failure probability is highly desirable in any mobile cellular communication system. Recently, several handover schemes have been proposed to do this $[1-4]$. At present, all handover requests to a base station from mobiles of the adjacent cells are enqueued in a common queue, and processed based on some prioritisation criteria and the availability of free

(i) If a free channel is available in cell $j$, it is assigned to the mobile; this results in handover success.

(ii) If a free channel is not available in cell $j$ and $\mathrm{Q}$,, is empty, then the handover request is enqueued in $\mathrm{Q}_{j i}$.

(iii) If a free channel is not available in cell $j$ and $\mathrm{Q}$, is nonempty, then the mobile is made to exchange its channel with the channel held by the mobile whose handover request has the highest priority in $\mathrm{Q}_{i j}$.

Simulation model: The performance evaluation of the new scheme is carried out in this Letter by considering the call traffic in two adjacent cells, each with 50 channels. This is sufficient to give a general indication of the comparative benefit of using handover channel exchanges. In each cell, the new calls are assumed to arrive at Poisson rate with a mean of $\lambda$. The mean interarrival time $(1 / \lambda)$ is varied to study the effect of increased offered traffic on the probabilities of call blocking, handover failure and call dropping. When a channel is assigned to a new call or a handover call, it is assumed that the channel is held by the call for a duration $t_{c}$, which is exponentially distributed with a mean of $1 / \mu(=$ $100 \mathrm{~s})$. The duration $t_{h}$ a mobile spends in the cell before requesting 


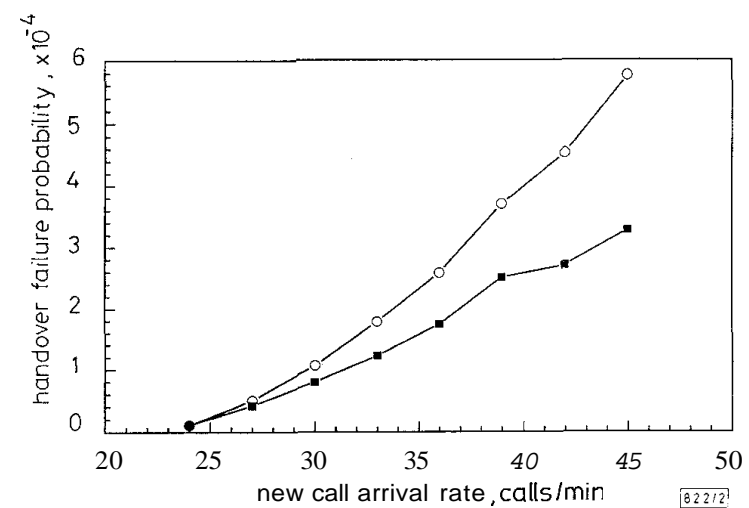

Fig. 2 Handoverfailure probability against new call arrival rate

without channel exchange

with channel exchange

a handover to the other cell is also asssumed to be exponentially distributed with a mean of $1 / \sigma(=60 \mathrm{~s})$. Thus, if $t_{c} \leq t_{h}$, the call is terminated within the current cell; otherwise, a handover request to the other cell is generated after holding the channel for a duration $t_{h}$ in the current cell. With this model, a call may result in several handovers between the two cells before it is either naturally terminated or forced to terminate owing to handover failure. The mobiles are assumed to move with uniform speed within the handover area, and the handover area traversal time is assumed to be normally distributed with a mean of 10 s and standard deviation of $2 \mathrm{~s}$. Thus, the radio power degradation within the handover area will be monotonic. The prioritisation of the handover requests is carried out as per the measurement based scheme [4].

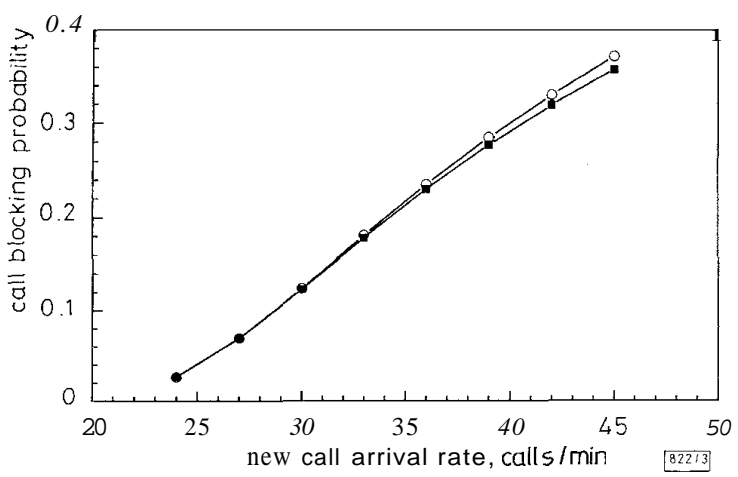

Fig. 3 Call blocking probability against new call arrival rate

$\mathrm{O}-\mathrm{O}$ without channel exchange

with channel exchange

Results and discussions: The above model was simulated using the Simscript II language. The number of new call requests simulated in each cell was one million. The simulation results calculated as averages for both the cells are shown in Figs. 2 - 4. The improvement in handover success rate caused by channel exchanges is clear from Fig. 2. As seen here, at a call traffic of $45 \mathrm{call} / \mathrm{min}$, the new scheme reduces handover failure probability to almost $50 \%$ of that without channel exchanges.

As is clear from Fig. 3, the new scheme does not degrade the call blocking rate as compared to the scheme without channel exchanges. It is interesting to note that at higher call arrival rates there is some reduction in call blocking probability. These reductions in call blocking probability are possible because, in the new scheme, a part of the handover traffic is managed with channel exchanges and, as a consequence, the probability of assignment of released channels to new calls increases.

The role of handover channel exchanges in increasing the number of successful calls is clear from Fig. 4. Call dropping is defined here as the failure of a call in progress owing to handover failure.

The superiority of the handover channel exchange scheme is clearly evident from the simulation results discussed above.

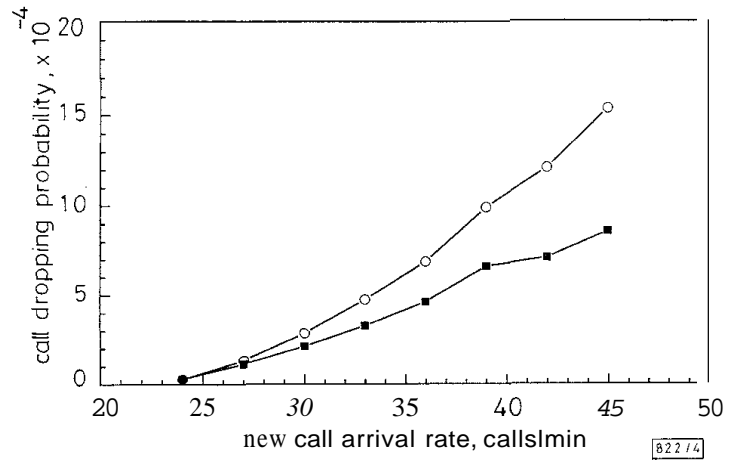

Fig. 4 Call dropping probability against new call arrival rate

without channel exchange

with channel exchange

Conclusion: The new handover scheme improves the handover success rate by exchanging channels between two mobiles moving in opposite directions across the handover area of adjacent cells. Performance evaluation using a two cell simulation model has shown that the new scheme not only minimises handover failure and call dropping probabilities but also reduces call blocking probability. These results clearly show the usefulness of the channel exchange scheme in modem mobile cellular communication systems.

\section{References}

1 ANVEKAR, D.K., AGRAWAL, P., and NARENDRAN, B.: 'A traffic-driven channel reservation scheme for handovers in mobile cellular networks". Proc. Sixth Int. Conf. Wireless Communications, Calgary, Alberta, Canada, July 1994,

2 ANVEKAR. D K., and AGRAWAL, P : 'A new criterion for processing handover requests in mobile cellular networks'. Proc IEEE Int. Conf. Personal Wireless Communications, India, August 1994

3 AGRAWAL. P, ANVEKAR, D.K., and NARENDRAN, B.: 'Minimizing cellular handover failures without channel utilization loss'. Proc. Globecom '94, San Fransisco, USA, 1994

4 TEKINAY. $\mathrm{s}$, and JABBARI. B.: 'A measurement-based prioritization scheme for handovers in mobile cellular networks', IEEE $J$. Sel. Areas Commun., 1992, 10, (8), pp. 1343-1350 